\title{
Influência da micropigmentação de sobrancelhas na autoestima de pacientes
}

\section{oncológicos}

\author{
Influence of eyebrow micropigmentation on the self-esteem of cancer patients \\ Influencia de la micropigmentación de las cejas en la autoestima de los pacientes con cáncer
}

Recebido: 02/12/2021 | Revisado: 08/12/2021 | Aceito: 14/12/2021 | Publicado: 21/12/2021

Ires Caroline Roque Costa

ORCID: https://orcid.org/0000-0002-2461-5080

Faculdade Independente do Nordeste, Brasil

E-mail: irescaroline0@gmail.com

Laís Xavier Rodrigues

ORCID: https://orcid.org/0000-0002-8643-3290

Faculdade Independente do Nordeste, Brasil

E-mail: laisx_rodrigues@outlook.com

Jeane Rocha Santos

ORCID: https://orcid.org/0000-0002-1398-3638

Faculdade Independente do Nordeste, Brasil

E-mail: jeane@fainor.com.br

\begin{abstract}
Resumo
O procedimento de dermopigmentação de sobrancelhas tem grande influência na autoestima em pacientes oncológicos, aliviando aspectos psicológicos que podem ter sido abalados por conta do severo tratamento de câncer e suas consequências. Dessa forma, a dermopigmentação de sobrancelhas é um procedimento importante, muito procurado por pacientes que buscam realizar essa conquista, no intuito de elevar a autoestima, além de renovar olhares, e sorrisos. Objetivo analisar a influência da micropigmentação de sobrancelhas na autoestima dos pacientes oncológicos. Foi realizada uma revisão de literatura, tendo como coleta de dados através de bases eletrônicas, tais como: a Scientific Eletronic Library Online - Scielo, Bireme, e Google acadêmico e LILACS Literatura científica e técnica da América Latina e Caribe/BVS - Biblioteca Virtual em Saúde. Os achados dessa pesquisa demonstram o impacto que a dermopigmentação tem na autoestima e melhora na qualidade de vida de pacientes oncológicos, trazendo também conhecimento aos leitores sobre a importância da dermopigmentação como um procedimento efetivo que pode satisfazer o aspecto físico e estético dos indivíduos. Conclui-se que a micropigmentação é um recurso eficiente para reconstruir a falha nas sobrancelhas de pacientes que perderam os fios devido o tratamento quimioterápico. Novos estudos acerca dos procedimentos são necessários e importantes.
\end{abstract}

Palavras-chave: Câncer; Dermopigmentação; Micropigmentação; Autoestima.

\begin{abstract}
The eyebrow dermopigmentation procedure has a great influence on self-esteem in cancer patients, alleviating psychological aspects that may have been affected by the severe treatment of cancer and its consequences. Thus, dermopigmentation of the eyebrows is an important procedure, much sought after by patients whon seek to achieve this achievement, in order to raise self-esteem, in addition to renewing looks and smiles. Objective to analyze the influence of eyebrow micropigmentation on the self-esteem of cancer patients. A literature review was carried out, with data collection through electronic databases, such as: Scientific Electronic Library Online - Scielo, Bireme, and Academic Google and LILACS Scientific and Technical Literature of Latin America and the Caribbean/VHL Library Virtual in Health. The findings of this research demonstrate the impact that dermopigmentation has on selfesteem and improvement in the quality of life of cancer patients, also bringing knowledge to readers about the importance of dermopigmentation as an effective procedure that can satisfy the physical and aesthetic of individuals. It is concluded that micropigmentation is an efficient resource to reconstruct the flaw in the eyebrows of patients whon lost hair due to chemotherapy treatment. New studies about the procedures are necessary and important.
\end{abstract}

Keywords: Cancer; Dermopigmentation; Micropigmentation; Self-esteem.

\section{Resumen}

El procedimiento de dermopigmentación de cejas tiene una gran influencia en la autoestima del paciente oncológico, aliviando aspectos psicológicos que pueden haber sido afectados por el tratamiento severo del cáncer y sus consecuencias. Así, la dermopigmentación de las cejas es un procedimiento importante, muy buscado por los pacientes que buscan lograr este logro, con el fin de elevar la autoestima, además de renovar miradas y sonrisas. Objetivo analizar la influencia de la micropigmentación de cejas en la autoestima de pacientes con cáncer. Se realizó una revisión de la literatura, con recolección de datos a través de bases de datos electrónicas, tales como: Biblioteca 
Científica Electrónica en Línea - Scielo, Bireme y Academic Google y LILACS Literatura Científica y Técnica de América Latina y el Caribe / BVS - Biblioteca Virtual en Salud. Los hallazgos de esta investigación demuestran el impacto que tiene la dermopigmentación en la autoestima y la mejora de la calidad de vida de los pacientes con cáncer, aportando también conocimiento a los lectores sobre la importancia de la dermopigmentación como un procedimiento eficaz que puede satisfacer las necesidades físicas y estéticas. de los individuos. Se concluye que la micropigmentación es un recurso eficiente para reconstruir la falla en las cejas de pacientes que perdieron cabello debido al tratamiento de quimioterapia. Nuevos estudios sobre los procedimientos son necesarios e importantes.

Palabras clave: Cáncer; Dermopigmentación; Micropigmentación; Autoestima.

\section{Introdução}

O câncer é uma doença crônica que pode gerar no individuo grandes impactos, e abala tanto a saúde física quanto a emocional. Muitos pacientes oncológicos precisam enfrentar, além das dores e sintomas advindos do câncer, um tratamento exaustivo e doloroso que pode vir até mesmo a causar certas deformidades físicas. Por consequência, o impacto gerado no psicológico e emocional destes pacientes também é relevante, problemas de baixa autoestima e depressão podem vir à tona (Cangussu, 2010).

A depressão é o transtorno psiquiátrico mais comum em pacientes com câncer, com prevalências variando de $22 \%$ a $29 \%$ (Bottino et al., 2009). Dessa forma, é necessária a atenção não apenas no quadro clínico de diagnóstico e ao seu tratamento, mas também a percepção das condições psicológicas. Um dos fatores resultantes do tratamento oncológico que suscita consequências psicológicas, além das físicas, é a queda de cabelo e pelos por toda região corpórea (Pisoni, 2013).

A perda dos cabelos é uma das maiores preocupações, pincipalmente entre mulheres, e pode ser o resultado de vários fatores: efeitos colaterais da quimioterapia, ansiedade, sensibilidade da pele ou de afeções cutâneas, afetando os pacientes em vários aspectos da vida (Rosa, 2020). De acordo com Giaretta (2016), algumas técnicas estéticas podem ser eficazes para ajudar na melhoria da autoestima dos pacientes com câncer, especialmente se tratando da perda dos fios das sobrancelhas, entre eles, a dermopigmentação.

A micropigmentação ou dermopigmentação, procedimento que pode ser realizado em pacientes oncológicos que já concluíram o tratamento, é uma alternativa para reconstruir os pelos da sobrancelha e recuperar a autoestima destes pacientes. A técnica baseia-se na utilização de pequenas agulhas, através de aparelhos como demógrafo ou tebori, que pode redesenhar os fios da sobrancelha (Giaretta, 2016). Esta técnica então se torna uma alternativa para melhorar a autoaceitação destes pacientes.

Diante do exposto, esta pesquisa tem como objetivo estudar a influência da micropigmentação de sobrancelhas na autoestima dos pacientes oncológicos.

\section{Metodologia}

Foi realizada uma revisão de literatura, que segundo Taylor e Procter (2002), tem como uma tomada de contas sobre o que foi publicado acerca de um tópico específico. Além disso, assume o caráter de uma pesquisa descritiva exploratória, já que se trata de um tema pouco estudado, ou explorado (Gil, 2010). A coleta de dados foi realizada através de bases eletrônicas, tais como: a Scientific Eletronic Library Online - Scielo, Bireme, Google acadêmico, LILACS Literatura científica e técnica da América Latina e Caribe/BVS - Biblioteca Virtual em Saúde. Inicialmente, foram selecionados 10 artigos que abordaram o tema deste estudo entre revisões de literatura e estudos de caso, entretanto 4 artigos foram descartados por não se enquadrarem totalmente nos critérios de inclusão e exclusão. Posteriormente, foi feita a leitura da revisão e da introdução dos artigos e como critérios de inclusão foram adotados: artigos que tivessem relação com a temática e artigos publicados entre os anos de 2005 a 2021; e como critérios de exclusão: não foram aceitos artigos que não tinham ligação com o tema e que tinham sido publicados anteriormente ao ano de 2005. A análise dos dados obtidos foi realizada através de uma ferramenta que possibilitou organizar 
os dados dos artigos em: título, autor e ano, tema, objetivo, métodos e resultados. Desta maneira ficou mais clara e objetiva a abordagem da temática. Também foi realizada uma análise descritiva dos dados obtidos correlacionando-os com o tema. O estudo seguiu os aspectos éticos dispostos na Lei de Direitos Autorais: Lei número 9.610, de fevereiro de 1998, assegurando que as literaturas que foram utilizadas no estudo são referenciadas corretamente, evitando assim, cópias e plágios.

\section{Resultados e Discussão}

Fizeram parte deste estudo 6 artigos que foram publicados entre 2014 a 2020, cujos resultados desvelavam os benefícios da micropigmentação das sobrancelhas em pacientes oncológicos. Criou-se um quadro que possibilitou organizar os dados dos artigos em: autor e ano, título, objetivos, métodos e resultados, conforme apresentado no Quadro 1.

Quadro 1 - Resumo analítico dos artigos estudados.

\begin{tabular}{|c|c|c|c|c|}
\hline $\begin{array}{c}\text { AUTO } \\
\text { R\ANO }\end{array}$ & TÍTULO & OBJETIVOS & MÉTODOS & RESULTADOS \\
\hline $\begin{array}{l}\text { Oliveira } \\
\text { et al. } \\
12020\end{array}$ & $\begin{array}{l}\text { Harmonização } \\
\text { facial e técnicas } \\
\text { na } \\
\text { micropigmentaç } \\
\text { ão de } \\
\text { sobrancelhas. }\end{array}$ & $\begin{array}{lr}\text { Compreender } & \text { melhor as } \\
\text { técnicas do visagismo, sua } \\
\text { história e } r \text { evolução, } \\
\text { associadas a r micro } \\
\text { pigmentação } & \text { de } \\
\text { sobrancelhas, } & \text { utilizando } \\
\text { técnicas para harmonização } \\
\text { facial. }\end{array}$ & $\begin{array}{l}\text { Trata-se de um estudo descritivo e } \\
\text { exploratório, de aspecto qualitativo, a } \\
\text { partir de uma revisão de literatura. }\end{array}$ & $\begin{array}{l}\text { A partir de pesquisas realizadas deste trabalho, } \\
\text { observou-se tamanha importância da micropigmentação } \\
\text { e seus benefícios, mas também notou-se a escassez de } \\
\text { pesquisas científicas realizadas sobre o assunto, e com } \\
\text { isso este artigo fica como incentivo para outros alunos } \\
\text { buscarem conhecimentos e aprofundarem sobre o tema. }\end{array}$ \\
\hline $\begin{array}{l}\text { Leite et } \\
\text { al. } \\
\backslash 2014\end{array}$ & $\begin{array}{l}\text { Avaliação da } \\
\text { autoestima em } \\
\text { pacientes } \\
\text { oncológicos } \\
\text { submetidos a } \\
\text { tratamento } \\
\text { quimioterápico. }\end{array}$ & $\begin{array}{l}\text { Avaliar a autoestima de } \\
\text { pacientes oncológicos } \\
\text { submetidos } \\
\text { quimioterapia. }\end{array}$ & $\begin{array}{l}\text { Estudo descritivo-analítico; transversal; } \\
\text { quantitativo. Participaram } 156 \text { pacientes } \\
\text { de uma unidade de oncologia de um } \\
\text { hospital geral de médio porte. }\end{array}$ & $\begin{array}{l}\text { Encontrou-se maior frequência de pacientes que } \\
\text { apresentaram autoestima alta, sendo que alguns deles } \\
\text { apresentaram autoestima média ou baixa. A escala } \\
\text { apresentou um valor alfa de Cronbach de } 0,746 \text {, } \\
\text { considerando-se sua consistência interna boa e aceitável } \\
\text { para os itens avaliados. Nenhuma variável independente } \\
\text { apresentou associação significativa com a autoestima. }\end{array}$ \\
\hline $\begin{array}{c}\text { Ferreira } \\
\backslash 2016\end{array}$ & $\begin{array}{l}\text { Qualidade de } \\
\text { vida, imagem } \\
\text { corporal e } \\
\text { satisfação nos } \\
\text { tratamentos } \\
\text { estéticos. }\end{array}$ & $\begin{array}{l}\text { Analisar a influência dos } \\
\text { tratamentos estéticos na } \\
\text { qualidade de vida, imagem } \\
\text { corporal e satisfação das } \\
\text { mulheres. }\end{array}$ & $\begin{array}{l}\text { Trata-se de um estudo observacional, } \\
\text { analítico, de corte transversal e de caráter } \\
\text { quantitativo, realizado em uma clínica de } \\
\text { estética localizada em Vitória da } \\
\text { Conquista, Bahia. }\end{array}$ & $\begin{array}{l}\text { A comparação das médias antes e depois do tratamento } \\
\text { mostra que para todos os domínios, houve uma } \\
\text { diferença significativa. Contudo, os tratamentos } \\
\text { estéticos melhoram consideravelmente questões globais, } \\
\text { físicas, psicológicas, relações sociais, imagem corporal } \\
\text { e o grau de satisfação, influenciando positivamente } \\
\text { tanto na imagem corporal quanto na qualidade de vida, } \\
\text { aumentando o grau de satisfação das mulheres. }\end{array}$ \\
\hline $\begin{array}{c}\text { Siviero } \\
\backslash 2016\end{array}$ & $\begin{array}{c}\text { Avaliação da } \\
\text { satisfação de } \\
\text { mulheres com } \\
\text { dermopigmenta } \\
\text { ção de } \\
\text { sobrancelhas } \\
\end{array}$ & $\begin{array}{l}\text { Analisar o nível de } \\
\text { satisfação } \\
\text { dermopigmentaçãode } \\
\text { sobrancelhas de estudantes } \\
\text { da Univali-BC. }\end{array}$ & $\begin{array}{c}\text { Pesquisa quantitativa baseada em um } \\
\text { levantamento de dados, sendo necessária } \\
\text { para a coleta de dados a realização de um } \\
\text { questionário com questões fechadas com } \\
24 \text { mulheres que apresentam } \\
\text { dermopigmentação de sobrancelhas. }\end{array}$ & $\begin{array}{l}\text { A grande maioria das pesquisadas encontra-se } \\
\text { satisfeitas com o resultado final de seu procedimento de } \\
\text { dermopigmentação bem como a qualidade da } \\
\text { profissional que lhe prestou atendimento. }\end{array}$ \\
\hline $\begin{array}{l}\text { Silva et } \\
\text { al. } \\
\backslash 2018\end{array}$ & $\begin{array}{l}\text { Micropigmentaç } \\
\text { ão de } \\
\text { sobrancelha: um } \\
\text { estudo da sua } \\
\text { evolução e } \\
\text { aplicação. }\end{array}$ & $\begin{array}{l}\text { Estudar a evolução da } \\
\text { micropigmentação } \\
\text { sobrancelhas e de } \\
\text { aplicação. }\end{array}$ & $\begin{array}{c}\text { Caracteriza-se por um estudo qualitativo, } \\
\text { do tipo descritivo exploratório, sua } \\
\text { pesquisa foi realizada através de revisão } \\
\text { bibliográfica em livro de } \\
\text { micropigmentação, publicação periódica, } \\
\text { revistas da área e sites da internet que } \\
\text { abordem o tema. }\end{array}$ & $\begin{array}{l}\text { Este estudo demonstrou que a técnica sofreu grande } \\
\text { evolução ao longo dos anos, e na sua forma de } \\
\text { aplicação. }\end{array}$ \\
\hline $\begin{array}{l}\text { Ribeiro } \\
\text { et al. } \\
\backslash 2020\end{array}$ & $\begin{array}{c}\text { Impacto da } \\
\text { Dermopigmenta } \\
\text { ção na } \\
\text { Autoestima de } \\
\text { Pacientes } \\
\text { Oncológicos } \\
\text { Submetidos ao } \\
\text { Tratamento } \\
\text { Quimioterápico }\end{array}$ & $\begin{array}{l}\text { Demonstrar os benefícios } \\
\text { da dermopigmentação nas } \\
\text { sobrancelhas na autoestima } \\
\text { de pacientes oncológicos } \\
\text { que se submeteram ao } \\
\text { tratamento quimioterápico. }\end{array}$ & $\begin{array}{l}\text { A metodologia utilizada se baseou em um } \\
\text { questionário semiestruturado } \\
\text { desenvolvido com questões relacionadas à } \\
\text { vivência durante o tratamento e após } \\
\text { realização do procedimento de } \\
\text { micropigmentação. }\end{array}$ & $\begin{array}{l}\text { Este estudo demonstrou que a técnica aplicada gera } \\
\text { efeito positivo na autoestima dos indivíduos com } \\
\text { câncer. Constatou-se também a necessidade de } \\
\text { publicações de mais artigos científicos sobre esse } \\
\text { assunto. }\end{array}$ \\
\hline $\begin{array}{l}\text { Martins } \\
\text { et al. } \\
12020\end{array}$ & $\begin{array}{l}\text { A Importância } \\
\text { dos } \\
\text { Procedimentos } \\
\text { Estéticos na } \\
\text { Autoestima da } \\
\text { Mulher }\end{array}$ & $\begin{array}{l}\text { Analisar o impacto de } \\
\text { procedimentos estéticos, } \\
\text { em especial, do design de } \\
\text { sobrancelha na autoestima } \\
\text { da mulher. }\end{array}$ & $\begin{array}{l}\text { Trata-se de um estudo de revisão de } \\
\text { literatura, realizado no período de } 15 \text { de } \\
\text { agosto a } 15 \text { de novembro de } 2020 \text {. }\end{array}$ & $\begin{array}{l}\text { Os achados dessa pesquisa demonstram que os } \\
\text { procedimentos estéticos aumentam a autoestima e dão } \\
\text { autoconfiança aos indivíduos que optam por esse tipo de } \\
\text { tratamento. }\end{array}$ \\
\hline
\end{tabular}

Fonte: Autores. 
De acordo com Sales et al. (2013), o diagnóstico de câncer mamário provoca nas mulheres grandes impactos emocionais, destacando-se o medo do diagnóstico, a realização de um procedimento cirúrgico, a incerteza dos possíveis resultados, os reaparecimentos dos efeitos colaterais do tratamento, a dor e todo o sofrimento por ela causados e saber que existe a possibilidade de morte. Além disso, ainda existem outros efeitos que acarretam a baixa autoestima dessas mulheres, tais como a queda do cabelo e dos fios das sobrancelhas (Oliveira et al., 2019).

Como uma solução para o problema, Fonseca e Tozo (2017) destacam que a micropigmentação torna-se uma alternativa para corrigir a falta dos pelos, uma vez que se trata de uma técnica de maquiagem, porém permanente, cujo objetivo é promover o embelezamento e melhorar a estética, principalmente do público feminino. O estudo realizado por Ribeiro et al (2020) também mostrou que a micropigmentação é uma das alternativas possíveis para reconstruir o desenho das sobrancelhas perdidas durante o tratamento para o câncer. Os pacientes oncológicos que realizaram a técnica relataram que se sentiram satisfeitos com o resultado do procedimento realizado.

A micropigmentação é um procedimento minucioso que exige do profissional um profundo conhecimento sobre diversos assuntos que envolvem a sua prática, como o entendimento sobre o sistema tegumentar, fisiologia do corpo humano e cosmetologia. O pigmento precisa ser depositado na camada correta da pele sem gerar traumas ao tecido, as formulações utilizadas precisam ser específicas para cada indivíduo caso apresente alguma patologia. Por essa razão para Flores (2016) a micropigmentação é um procedimento que deve ser realizado por um profissional da área de estética, que entenda sobre a sua execução e sobre a importância da biossegurança dos materiais utilizados.

O fator estético tem grande relevância no que se refere a questão da autoestima, isso porque estão em constante conexão. Uma vez que a autoestima está elevada, a parte psíquica também está sendo favorecida, sendo a afirmação oposta também verdadeira. Com isso, Ferreira et al (2016), busca estudar a influência dos procedimentos estéticos no bem-estar, qualidade de vida, satisfação da imagem corporal e pessoal.

Corroborando com o artigo supracitado está o estudo de Martins et al. (2020) que evidenciou, por meio de uma revisão de literatura, que os procedimentos estéticos são capazes de elevar a autoestima e de melhorar o convívio social dos indivíduos. Destacando que as intervenções estéticas, incluindo a micropigmentação e design de sobrancelhas também aprimoram consideravelmente questões coletivas, físicas, psicológicas, satisfação da imagem pessoal, relacionamentos sociais, contribuindo de maneira positiva tanto no contentamento da imagem corporal, quanto no bem-estar e qualidade de vida, elevando o grau de satisfação dos indivíduos que optam por esse tipo de tratamento.

A micropigmentação de sobrancelhas é um procedimento que auxilia não apenas a parte estética, mas também no quesito psíquico que foi abalado durante todo o procedimento, desde a descoberta da neoplasia até o tratamento que possui diversos efeitos colaterais, Leite et al (2014) avalia em sua pesquisa a autoestima de pacientes oncológicos em fase de tratamento quimioterápico, classificando-a em baixa, média, e alta, o resultado apresentado a partir dos itens avaliados considera que os pacientes oncológicos possuem sua autoestima dentro do aceitável.

A autoestima caracteriza a identidade dos indivíduos e está relacionada diretamente ao psíquico, assim como com a satisfação pessoal e aceitação no âmbito social. Silveiro (2016) enfatiza que a micropigmentação renova a autoconfiança em mulheres que buscam a evidenciação da personalidade feminina, gerando também uma identificação e satisfação com o profissional que realizou o procedimento. O paciente deve estar apto para realizar o procedimento para que não ocorra nenhuma intercorrência, por esse motivo é feito uma avaliação prévia com o preenchimento de uma ficha de anamnese. E em pacientes oncológicos é necessária uma autorização médica para verificar se o mesmo possui imunidade adequada para o procedimento. O pigmento utilizado é visto como um antígeno ao corpo humano, segundo Jesus (2002) o sistema imune irá elaborar respostas imunes que destruirão as células que portam tais antígenos. Este fenômeno é chamado de vigilância 
imunológica, e é realizado pelas células efetoras imunológicas, tais como linfócitos T, macrófagos e células matadoras naturais.

O procedimento de micropigmentação de sobrancelhas atua na melhoria da condição de vida de muitas mulheres trazendo harmonia ao rosto e reestabelecendo o bem-estar, entretanto, a dermopigmentação vai além da reconstrução somente de sobrancelhas. Por exemplo, o paciente oncológico que foi submetido à mastectomia pode através desse procedimento realizar a pigmentação areolar, também chamada de micropigmentação paramédica, que segundo Sanderson et al., (2009) e Souza (2015) é capaz de melhorar o desconforto pela falta de beleza, reconstruindo um design areolar diante das cicatrizes deixadas pela cirurgia, promovendo a essas pacientes autoestima e principalmente uma nova oportunidade de recomeço.

Vale ressaltar também que, além de todos os fatores mencionados anteriormente, o procedimento de micropigmentação vem sofrendo constantes mudanças ao longo dos anos, tanto no seu estudo, quanto na sua aplicação. Segundo Silva et al (2018), a técnica vem evoluindo cada vez mais, o que antes era tratado como um procedimento definitivo, com efeito tatuagem, hoje se apresenta em diversos métodos e capazes de atender a todos os gostos, desde sobrancelhas com a arquitetura dos fios mais delicados e naturais, como a nanoblading, até sobrancelhas mais marcantes e evidentes, como as realizadas com as técnicas de shadow ou shadowline.

Levando em consideração o processo de evolução da técnica de micropigmentação, Oliveira et al (2020) busca em sua pesquisa compreender melhor as técnicas do visagismo, sua história e mudanças. Com isso, nota-se que a micropigmentação dos dias atuais é de fato efetiva, conseguindo produzir resultados de excelência, suas variadas técnicas atendem as expectativas de todo indivíduo que busca por um preenchimento natural e harmônico das sobrancelhas, sobretudo para pacientes oncológicos que sofreram com a perda capilar.

\section{Conclusão}

Este estudo demonstrou por meio de uma revisão de literatura que o procedimento de micropigmentação é uma das principais alternativas para reconstruir o desenho das sobrancelhas cujos pelos foram perdidos no tratamento quimioterápico. Por se localizarem na face e por serem consideradas essenciais para a harmonia dessa região, perder as sobrancelhas pode vir a causar danos a autoimagem do indivíduo.

Com o objetivo de diminuir o sofrimento provocado pelo câncer, a reconstrução das sobrancelhas torna-se um recurso útil, capaz de melhorar a qualidade de vida destes pacientes. Comprovando que a micropigmentação é capaz de elevar a autoestima, reestabelecendo a autoconfiança uma vez perdida com a ausência dos fios naturais.

Diante disso, acredita-se que a realização de pesquisas relacionadas ao tema sejam fundamentais para que os profissionais possam conhecer e aprimorar a técnica, ajudando ainda mais indivíduos. Sugere-se que os futuros estudos abordem como a micropigmentação de sobrancelhas deve ser realizada, descrevendo protocolos mais seguros.

\section{Referências}

Bottino, S. M. B., \& Fráguas, R., \& Gattaz, W. F. (2009). Depressão e câncer. Archives of Clinical Psychiatry. 36, 109-115.

Cangussu, R. D. O., \& Soares, T. B. D. C., \& Barra, A. D. A., \& Nicolato, R. (2010). Sintomas depressivos no câncer de mama: Inventário de Depressão de Beck-Short Form. Jornal Brasileiro de psiquiatria, 59, 106-110.

Ferreira, J. B., \& Lemos, L. M. A., \& Da Silva, T. R. (2016). Qualidade de vida, imagem corporal e satisfação nos tratamentos estéticos. Revista Pesquisa em Fisioterapia, 6(4), 402-410.

Fonseca, P. F. B., \& Tozo, F. de. C. (2017) Micropigmentação de sobrancelhas. Curitiba. <https://docplayer.com.br/51489381-Micropigmentacao-de17 sobrancelhas-piter-felipe-brogian-da-fonseca-1-fabiana-de-cassia-tozo-2.html>.

Flores, F. (2016). Saiba tudo sobre: micropigmentação das areolas mamárias X tatuagem. <https://www.quimioterapiaebeleza.com.br/saiba-tudo-sobremicropigmentacao-das-areolasmamarias-X-tatuagem-3d/>. 
Research, Society and Development, v. 10, n. 17, e85101724290, 2021

(CC BY 4.0) | ISSN 2525-3409 | DOI: http://dx.doi.org/10.33448/rsd-v10i17.24290

Giaretta, E. (2016). Dermopigmentação/Arte e Responsabilidade (4a ed.). Academia Brasileira de Micropigmentação.

Gil, A. C. (2010). Como elaborar projetos de pesquisa (10a ed.). Atlas.

Leite, M. A. C. (2014). Avaliação da autoestima em pacientes oncológicos ao tratamento quimioterápico. Dissertação (Mestrado em Enfermagem) - Alfenas: Universidade Federal de Alfenas.

Jesus, M. C. D. (2002). Imunologia do câncer. https://www.ciencianews.com.br/index.php/publicacoes/artigos-cientificos/imunologia-do-cancer/> .

Martins, R. D. S. G., \& Ferreira, Z. A. B. (2020). A Importância dos Procedimentos Estéticos na Autoestima da Mulher/The Importance of Aesthetic Procedures in Women's Self-Esteem. ID on line Revista De Psicologia, 14(53), 442-453. 10.14295/idonline.v14i53.2807.

Oliveira, T. R., \& Corrêa, C. S. L., \& Weiss, V. F., \& Baquião, A. P. D. S. S., \& Carvalho, L. L., \& Grincenkov, F. R. S., \& Carvalho, S. M. (2019). Câncer de mama e imagem corporal: impacto dos tratamentos no olhar de mulheres mastectomizadas. Saúde e Pesquisa, 12(3), 451-462). 10.17765/21769206.2019v12n3p451-462.

Oliveira, H. S., \& Gonçalves, T. O., \& Faria, W. J. J. (2020). Harmonização facial e técnicas na micropigmentação de sobrancelhas.http://repositorio.aee.edu.br/bitstream/aee/9536/1/HARMONIZA\%C3\%87\%C3\%83O\%20FACIAL\%20E\%20T\%C3\%89CNICAS\%20NA\%20M ICROPIGMENTA\%C3\%87\%C3\%830\%20DE\%20SOBRANCELHAS.pdf>.

Pisoni, A. C. (2013). Dificuldades vivenciadas por mulheres em tratamento para o câncer de mama. Revista de Pesquisa: Universidade Federal do Estado do Rio de Janeiro - Online, 5(3), 194-201.

Ribeiro, I. R., Ferreira, Z. A. B., Maturano, A. S. S., \& de Matos Mendes, A. M. F. (2020). Impacto da Dermopigmentação na Autoestima de Pacientes Oncológicos Submetidos ao Tratamento Quimioterápico. Rev. De Psicologia. 14(50), 1060-1070.

Rosa, D. J. F. D. (2020). Moda inclusiva: o uso de turbantes na rotina de mulheres em tratamento contra o câncer. https://repositorio.ifsc.edu.br/handle/123456789/1636.

Sales, C. A. C. C., \& Paiva, L., Scandiuzzi, D., \& Anjos, A. C. Y. (2013). Qualidade de vida de mulheres tratadas de câncer de mama: funcionamento social. Revista brasileira de cancerologia, 47(3), 263-272.

Silva, A. C., \& Wanka, A., \& Gallas, J. C., \& Paganini, T. (2018). Micropigmentação de sobrancelha: um estudo da sua evolução e aplicação. Curso Superior de Tecnologia em Cosmetologia e Estética. Balneário Camboriú: Universidade do Vale do Itajaí.

Siviero, C. B., \& Custódio, M. C., \& Marcuzzo, M. (2016). Avaliação da satisfação de mulheres com dermopigmentação de sobrancelhas. Trabalho de Iniciação Científica do Curso Superior de Tecnologia em Cosmetologia e Estética. Balneário Camboriú: Universidade do Vale do Itajaí.

Sanderson, B. F., \& Bitencourt, C. F., \& Silva, F. F., \& Balconi, G. T., \& Brito, T. L. C., \& Duarte, M. M. F. (2009). Dermopigmentação uma alternativa estética e reparadora. ULBRA.

Souza, V. A. \& Mejia, D. (2015). Benefícios da micropigmentação paramédica em mulheres mastectomizadas. Monografia de pós graduação em Estética e Cosmetologia da Faculdade de Tecnologia do Ipê de Manaus-AM.

Taylor, D., \& Procter, M. (2021). The literature review: a few tips on conducting it. In: https://guides.library.pdx.edu/ld.php?content_id=10938141. 